\title{
Pregnancy is associated with psychiatric symptoms in a low-income countryside community of Brazil
}

This article was published in the following Dove Press journal:

Neuropsychiatric Disease and Treatment

29 November 201I

Number of times this article has been viewed

\section{Fernando MV Dias ${ }^{1-3}$ \\ Claudio SD Junior ${ }^{4}$ \\ Glaura C Franco ${ }^{5}$ \\ Antônio L Teixeira ${ }^{3}$ \\ Angela M Ribeiro ${ }^{2}$}

'Department of Medicine and Nursing, Federal University of Viçosa, Viçosa;

${ }^{2}$ Graduate Program in Neurosciences,

Laboratory of Molecular and

Behavioral Neuroscience ( $\mathrm{LaNeC}$ );

${ }^{3}$ Graduate Program in Neurosciences,

Neuropsychiatric Branch Research;

${ }^{4}$ Department of Sociology and

Anthropology; ${ }^{5}$ Department of

Statistics, Federal University of Minas

Gerais, Belo Horrizonte, Brazil
Correspondence: Fernando Machado Vilhena Dias

Department of Medicine and Nursing, Federal University of Viçosa, Avenida PH Rolfs s/n, Campus Universitário, CEP 3657I-000, Viçosa, Minas Gerais, Brazil Tel +5531 38993906

Fax +553138993906

Email fernandomvdias@hotmail.com
Background: Psychiatric symptoms during pregnancy induce an increase in morbidity and also in the mortality levels among women and children. However, the real association between pregnancy and psychiatric disorders and the peculiarities of the phenomenology of symptoms in underprivileged countryside communities remain uncertain.

Objective: To verify the association between psychiatric disorder, symptoms, and pregnancy among women from a low-income countryside community and to determine the specific cutoff points for major depression diagnosis according to Beck Depression Inventory for the different trimesters of pregnancy in this population.

Methods: Ninety-four pregnant women and 38 healthy women from the Conceição do Mato Dentro health service, a rural low-income community in Brazil, participated in the present study. Psychiatric examination included a structured clinical interview for psychiatric disorders according to Mini International Neuropsychiatric Interview and psychometric scales such as the Yale-Brown Obsessive Scale, the Beck Depression Inventory, and the Hamilton Anxiety Scale. The cutoff points for Beck Depression Inventory were determined through the application of receiver operating characteristic curves considering the diagnosis of major depression according to Mini International Neuropsychiatric Interview.

Results: The pregnant women had a higher frequency of psychiatric disorders and depression and anxiety symptoms. All cutoff points of Beck Depression Inventory were equal or higher than 12 with high sensitivity and specificity. Although the modified cutoff was selected based on both high sensitivity and high specificity, they were lower than when the cutoff was applied to nonpregnant women.

Conclusion: Pregnancy was associated with the occurrence of psychiatric disorders and depressive and anxiety symptoms. In comparison to the literature, the present results indicate that there are different cutoff points in the Beck Depression Inventory for pregnant women from different cultures and in different pregnancy trimesters.

Keywords: pregnancy, psychiatric symptoms, low-income countryside communities

\section{Introduction}

Psychiatric symptoms during pregnancy induce an increase in morbidity and also in the mortality levels among women and children. Fetal mortality suffers an increase among mothers with mental disorders: stillbirths occur 1.1 times more in pregnant women with schizophrenia and twice more in those who present affective disease diagnoses, such as major depression and bipolar disorder. ${ }^{1}$ Anxiety symptoms experienced by the mother related to pregnancy may alter the child's neurodevelopment, leading to important brain region atrophies such as the prefrontal cortex. ${ }^{2}$ 
The current literature results are not accurate as to whether (1) pregnancy itself creates a condition that raises the risk of mental disturbance development, such as affective and psychotic disorders, (2) pregnant women show no difference when compared to the general population, and (3) pregnancy can represent a protective factor for mental disturbances. ${ }^{3,4}$ Additionally, exposure to other risk factors, such as being an immigrant woman, could strongly increase the development of psychiatric disturbances. ${ }^{5}$

There is a gap in the literature about epidemic studies that systematically investigate the psychiatric symptoms in pregnant women from the countryside of developing countries. ${ }^{6,7}$ In one of the few studies found in the literature, it was observed that in that countryside population the prevalence rates of major depression may affect up to $25 \%$ of pregnant women. ${ }^{8}$ Thus, the evidence that belonging to lowincome countryside communities increases the probability of developing psychiatric symptoms during pregnancy is still uncertain.

Therefore, the evaluation of pregnant women who belong to countryside communities can contribute to the clarification of the particularities of psychiatric manifestations in this group of people, as well as to help comprehend the relationship between environmental risk factors and fetal brain function alterations. ${ }^{9,10}$

In the present study, the aims were to verify the association between pregnancy and psychiatric disorders and symptoms in women from a low-income countryside community and the determination of specific cutoff points of this population to major depression diagnosis according to Beck Depression Inventory (BDI).

\section{Methods}

Ninety-four pregnant women and 38 healthy women from the Conceição do Mato Dentro health service, a rural low-income community in Brazil, were included in the present study. Among the pregnant women, 17 were in the first trimester (0-12 weeks), 39 were in the second trimester (12-24 weeks), and 38 were in the third trimester.

The estimated population of Conceição do Mato Dentro was 18,558 in 2008 . Its territory measures approximately $1700 \mathrm{~m}^{2}$. Most of the population live in the countryside and rely on low agricultural productivity, which reflects a medium human development index. The district does not have a proper medical service infrastructure and, very often, pregnant women are sent to other health centers to give birth.

All pregnant women who underwent prenatal exams in the district ambulatory between July 2009 and December 2009, and agreed to take part in the research, were included. Antipsychotics and antidepressant medication intake, mental retardation from a previous diagnosis, neurosurgery history, cranial traumas, antibiotics, corticoids, and anti-inflammatory intake, infections, neurodegenerative disorders, and autoimmune diseases within the last few weeks were taken as exclusion criteria.

The control group was formed by women who visited the local health care centers between July 2009 and December 2009 and had to undergo examination in order to prevent cervical cancer. This prevention policy, which advises women to take the medical examination once a year, is adopted all over the country. These women were invited to take part in the research. They had to be between 18 and 35 years old. The same exclusion criteria adopted for pregnant women was applied to the control group. Subjects who met all inclusion criteria and accepted to participate were included in the study.

Written informed consent was obtained from all subjects. The study was approved by the local ethics committee (process number ETIC 05950203000-09).

Pregnant women and control subjects underwent psychiatric examinations, which included a structured clinical interview for current psychiatric disorders according to Mini International Neuropsychiatric Interview (MINI), and psychometric scales, such as the Yale-Brown Obsessive Scale, BDI, and Hamilton Anxiety Scale (HAS). ${ }^{11-13}$

The Kolmogorov-Smirnov test was used to verify the Gaussian assumption for the variables under study at a 0.05 significance level. The distribution for all variables was not normal, except for age and schooling period.

Comparison between the pregnant women group and control group was performed using a two-tailed Student's $t$-test for continuous variables with normal distribution. For continuous variables with nonnormal distribution, the Mann-Whitney U test was used. Comparisons for categorical variables between groups were performed by the chi-square test. The analyses were carried out using a two-tailed test at the 0.05 significance level.

To identify the cutoff points of the BDI for the sample used in the present study, sensitivity and specificity measures were used to distinguish pregnant women and controls with or without major depressive disorder based on MINI. Receiver operating characteristic (ROC) analysis was used to optimize the cutoff point. A ROC space is defined by the false-positive rate and the true-positive rate as $\mathrm{x}$-axis and $\mathrm{y}$-axis, respectively, depicting relative tradeoffs between true positives and false positives. As the x-rate is equivalent to sensitivity and 
the y-rate to specificity, the modified cutoff was selected based on both high sensitivity and high specificity. A value of the curve area over 0.7 was considered an adequate test.

Statistical analysis was performed using the SPSS version 17.0 software (IBM Corporation, Armonk, NY).

\section{Results}

Pregnant women group and control group did not differ in age, educational level, monthly income per capita, or when pregnancy occurred for the first time. Despite the existence of two outliers in the pregnant group, considering the monthly income per capita, there was no difference when the test was run again without outliers. The group of pregnant women presented a higher frequency of marriage than those in the control group $(P=0.003)$ (Table 1$)$.

No pregnant woman was under psychiatric treatment with antidepressants or antipsychotics, and so, none of them was excluded for this reason.

It was observed that pregnant women presented more current psychiatric disorders according to MINI $(P=0.04)$ (Table 2). The most frequent psychiatric disorders in pregnant women were: present major depression 16\%, general anxiety disorder 16\%, and alcohol and marijuana abuse 7.4\%. In control group, the results were: past major depression 10.5\%, present major depression $7.9 \%$, and general anxiety disorder $7.9 \%$. However, none of the disorders reached statistically significant difference when compared singly.

Table I Demographic features of pregnant women and control group

\begin{tabular}{|c|c|c|c|}
\hline & $\begin{array}{l}\text { Control } \\
(n=38)\end{array}$ & $\begin{array}{l}\text { Pregnant } \\
\text { women } \\
(\mathrm{n}=94)\end{array}$ & $P$ value \\
\hline \multicolumn{4}{|l|}{ Age (years) $^{a}$} \\
\hline $\begin{array}{l}\text { Mean } \pm \text { standard deviation } \\
\text { (median) }\end{array}$ & $\begin{array}{l}25.84 \pm 3.9 \\
(26)\end{array}$ & $\begin{array}{l}25.1 \pm 6.7 \\
(25)\end{array}$ & 0.45 \\
\hline \multicolumn{4}{|l|}{ Marital status (married) } \\
\hline Frequency & $20(52.6 \%)$ & $74(78.7 \%)$ & 0.003 \\
\hline \multicolumn{4}{|l|}{ Educational level (years) ${ }^{a}$} \\
\hline $\begin{array}{l}\text { Mean } \pm \text { standard deviation } \\
\text { (median) }\end{array}$ & $\begin{array}{l}10.5 \pm 3.22 \\
(11)\end{array}$ & $\begin{array}{l}9.03 \pm 4.3 \\
(9)\end{array}$ & 0.058 \\
\hline \multicolumn{4}{|c|}{$\begin{array}{l}\text { Monthly income per capita } \\
\text { (Brazilian reais) }^{\mathrm{c}, \mathrm{d}}\end{array}$} \\
\hline $\begin{array}{l}\text { Mean } \pm \text { standard deviation } \\
\text { (median) }\end{array}$ & $\begin{array}{l}257.3 \pm|4| .3 \\
(236)\end{array}$ & $\begin{array}{l}417.4 \pm 557.9 \\
(205)\end{array}$ & 0.72 \\
\hline \multicolumn{4}{|l|}{$\begin{array}{l}\text { Age when pregnancy } \\
\text { occured for the first time } \\
\text { (years) }^{c, e}\end{array}$} \\
\hline $\begin{array}{l}\text { Mean } \pm \text { standard deviation } \\
\text { (median) }\end{array}$ & $\begin{array}{l}19.4 \pm 3.12 \\
(19)\end{array}$ & $\begin{array}{l}20.8 \pm 4.5 \\
(20)\end{array}$ & 0.25 \\
\hline
\end{tabular}

Table 2 Comparison of current psychiatric disorders, according to Mini International Neuropsychiatric Interview and scores in psychometric scales, in pregnant women and control group

\begin{tabular}{|c|c|c|c|}
\hline & $\begin{array}{l}\text { Control } \\
(n=38)\end{array}$ & $\begin{array}{l}\text { Pregnant } \\
\text { women } \\
(n=94)\end{array}$ & $P$ value \\
\hline \multicolumn{4}{|l|}{ MINI Plus } \\
\hline Frequency & 9 (23.7\%) & $4 \mathrm{l}(43.6 \%)$ & 0.04 \\
\hline \multicolumn{4}{|l|}{ BDI } \\
\hline $\begin{array}{l}\text { Mean } \pm \text { standard deviation } \\
\text { (median) }\end{array}$ & $\begin{array}{l}2.58 \pm 7.02 \\
(0)\end{array}$ & $\begin{array}{l}8.05 \pm 7.56 \\
(7)\end{array}$ & 0.00 \\
\hline \multicolumn{4}{|l|}{ HAS } \\
\hline $\begin{array}{l}\text { Mean } \pm \text { standard deviation } \\
\text { (median) }\end{array}$ & $\begin{array}{l}3.39 \pm 6.18 \\
(0)\end{array}$ & $\begin{array}{l}6.11 \pm 6.83 \\
(4)\end{array}$ & 0.03 \\
\hline
\end{tabular}

Abbreviations: HAS, Hamilton Anxiety Scale; BDI, Beck Depression Inventory; MINI, Mini International Neuropsychiatric Interview.

The BDI and HAS confirmed a high prevalence of anxiety and depression symptoms in pregnant women when compared to the subjects of the control group $(P$ value $=0.00$ and 0.03 , respectively). Regarding obsessive and compulsive symptoms, there was no statistically significant difference between control group and pregnant group (1/38 (2.6\%) in control group and 5/94 (5.3\%) in pregnant group; $P$ value $=0.7)$

The following BDI cutoff points were found for the total sample of pregnant women group (Figure 1) and for the first, second, and third trimester, respectively: (1) cutoff point of

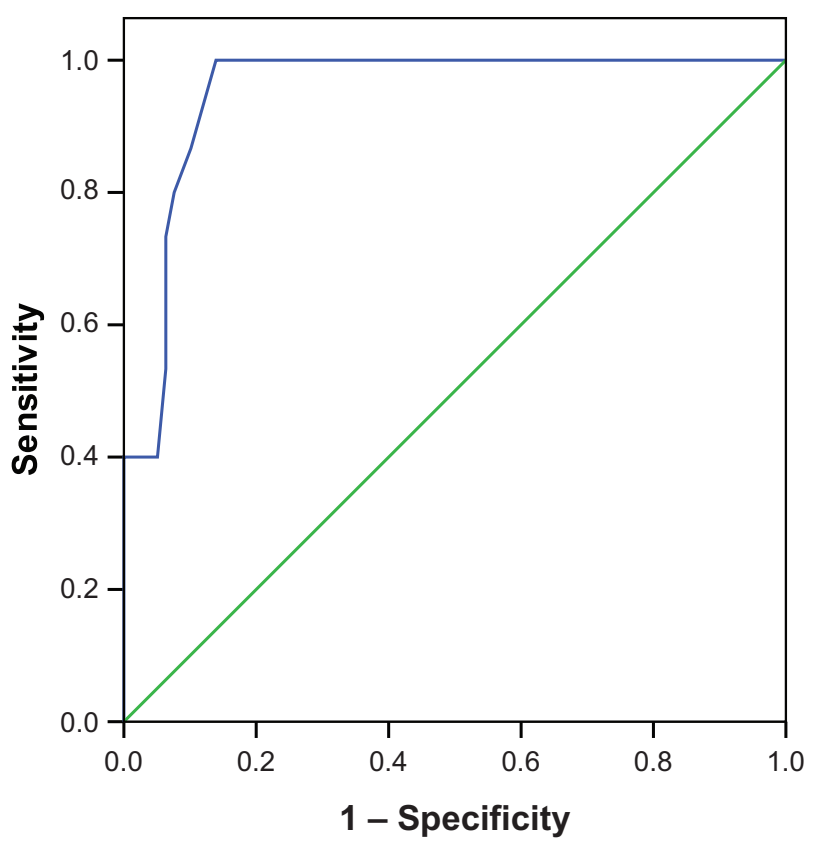

Figure I Receiver operating characteristic curve for determination of cutoff point of Beck Depression Inventory in total pregnant sample.

Note: Diagonal segments are produced by ties. 
12 (area under the curve $=0.95$, sensitivity $=95.2 \%$, and specificity $=85.6 \%$ ), (2) cutoff point of 13 (area under the curve $=0.96$, sensitivity $=100 \%$, and specificity $=93 \%$ ), (3) cutoff point of 12 (area under the curve $=0.95$, sensitivity $=93 \%$, and specificity $=83.4 \%$ ), and (4) cutoff point of 12 (area under the curve $=0.97$, sensitivity $=97 \%$, and specificity $=96 \%$ ). The differences in ROC curve among trimesters were not statistically significant. For the control group, the BDI cutoff was 10 (area under the curve $=0.99$, sensitivity $=100 \%$, and specificity $=99.4 \%$ ).

\section{Discussion}

A higher frequency of psychiatric disorders, according to current diagnosis, was observed among pregnant women compared to the control group in the present community. Fisher et al, studying the pregnancy effects on psychiatric symptoms in a similar population sample, found that medical assistance conditions were related to the emotional disturbance observed. ${ }^{14}$ Evidence of the poor quality of health care in this community is that none of the pregnant women were under psychiatric treatment. Even though no association was observed between the effects of pregnancy and specific demographic features of the population sample, the hypothesis that pregnancy represents a higher risk of psychiatric disorder occurrences in low-income countryside communities cannot be discarded. Other population features that were not assessed here, which represent a limitation of the present study, could also be a risk factor for pregnancy effects on psychiatric symptom manifestation. For instance, in a study of an urban Brazilian sample, Pereira et al showed that violence against women during pregnancy, history of accidents and catastrophes, and emotional or physical abuse during their lives were associated with major depression. ${ }^{15}$ Further studies would be necessary to clarify that question. The findings of the current study are consistent with other authors and support the statement that an efficient approach towards pregnant women who belong to low-income communities must be implemented in order to avoid maternal and childhood health damage. ${ }^{16,17}$ Moreover, as there is an association between neuropsychomotor development delay among children and transgenerational perpetuation of the poverty situation, the improvement of medical assistance to pregnant women could contribute to socially modifying these low-income communities. ${ }^{9}$

The higher frequency of anxiety and depressive symptoms observed in the pregnant women group compared to the control group is in accordance with results obtained by using MINI assessment regarding the prevalence of disorders.
According to the present results, pregnancy was not associated with obsessive-compulsive symptoms for that particular population. $^{18}$

However, some consideration must be given to the methods applied to assess anxiety and depressive symptoms. Despite the fact that HAS is a scale with widely confirmed validity, other tools that evaluate anxiety symptoms particularly related to pregnancy, such as the ten-item Pregnancy Anxiety Scale, may be more appropriate for such analyses. ${ }^{19,20}$ This scale includes issues such as the fear of abortion and the feelings related to delivery. ${ }^{20}$ To the authors' knowledge, up to the present time a comparison between HAS and the Pregnancy Anxiety Scale has not been used to evaluate diagnostic sensitivity and specificity. Considering pregnancy is a special period in the phenomenology of anxiety, the lack of a specific scale represents a possible limitation of the present study. ${ }^{21-23}$

Moreover, the use of the BDI scale to evaluate symptoms during pregnancy has been criticized by other authors. ${ }^{24}$ However, the Edinburgh Postnatal Depression Scale and the BDI scale are the most used with validity among obstetric populations, either during pregnancy or after birth. ${ }^{25-27}$ $\mathrm{Su}$ et al observed that BDI takes into consideration many somatic symptoms (five of 21) and, therefore, lacks the sensitivity to diagnose depression during pregnancy. ${ }^{28}$ In other words, the symptoms considered as depressive by the BDI may, in fact, be pregnancy physiological changes such as sexual desire, sleeping, and energy alterations. ${ }^{29,30}$ In future studies, a line of inquiry can be opened to understand mechanisms by which pregnancy influences the mood of pregnant women, which may enable a better understanding of brain function in conditions such as major depression. ${ }^{31}$ In spite of the high frequency of depressive symptoms among pregnant women who belong to this community, other studies that use different scales such as the Edinburgh Postnatal Depression Scale must be applied in order to confirm the results found in the present study. A specific scale for obsessive-compulsive disorder diagnosis could also be done, justifying the low frequency in this community. ${ }^{32}$

Tandon et al, unlike previous studies, found lower cutoff points in the BDI for major depression diagnosis in pregnant women when compared to cutoff points in the general population. ${ }^{33}$ In their study, a specific group of pregnant women (African Americans) was assessed. One of the possible explanations for the literature disagreement is that the cutoff points of the BDI vary according to the culture they are applied to. ${ }^{34}$ The present study, which was performed in a very peculiar community, presented a higher cutoff point of 12 , whereas the one marked for the general population 
is $10 .{ }^{35}$ Sensitivity and specificity are even worse when the lower cutoff is used in nonpregnant women. The same result for the general population was observed in the control group. The results obtained by the ROC curve during the three trimesters corroborate that for this pregnant population, the cutoff points with larger sensitivity and specificity were higher. It must be emphasized that during pregnancy evaluation, physiological and psychological changes do happen, which may differ for depression symptom manifestations in each trimester. ${ }^{36,37}$ Thereby, cutoff point evaluations in the different trimesters are of great importance for the best accuracy in diagnosis of major depression.

\section{Conclusion}

The present study confirms previous findings that pregnancy affects emotional aspects that can be detected as psychiatric symptoms. Pregnancy in low-income countryside populations seems to be associated with the occurrence of psychiatric disorders, such as anxiety and depressive symptoms. Nevertheless, different cutoff points for the BDI must be considered for pregnant women from different cultures and in different trimesters in order to achieve accurate diagnosis.

\section{Acknowledgments}

The authors thank Catherine Kelly Martins for revising the English editing. The present study was sponsored by Fundação de Amparo a Pesquisa do Estado de Minas Gerais (FAPEMIG, Brazil). The Brazilian funding agency has no role in study design, in the collection, analysis and interpretation of data, in the writing of the report, and in the decision to submit the article for publication.

\section{Disclosure}

The authors report no conflicts of interest in this work.

\section{References}

1. Brouwers EPM, van Baar AL, Pop VJM. Maternal anxiety during pregnancy and subsequent infant development. Infant Behav Dev. 2001; 24(1):95-106.

2. Buss C, Davis EP, Muftuler LT, Head K, Sandman CA. High pregnancy anxiety during mid-gestation is associated with decreased gray matter density in 6-9-year-old children. Psychoneuroendocrinology. 2010;35(1): 141-153.

3. Alder J, Fink N, Bitzer J, Hösli I, Holzgreve W. Depression and anxiety during pregnancy: a risk factor for obstetric, fetal and neonatal outcome? A critical review of the literature. JMatern Fetal Neonatal Med.2007;20(3): 189-209.

4. Vesga-López O, Blanco C, Keyes K, Olfson M, Grant BF, Hasin DS Psychiatric disorders in pregnant and postpartum women in the United States. Arch Gen Psychiatry. 2008;65(7):805-815.

5. Pinheiro SN, Laprega MR, Furtado EF. Psychiatric morbidity and alcohol use by pregnant women in a public obstetric service. Rev Saude Publica. 2005;39(4):593-598. Portuguese.
6. Rahman A, Iqbal Z, Bunn J, Lovel H, Harrington R. Impact of maternal depression on infant nutritional status and illness: a cohort study. Arch Gen Psychiatry. 2004;61(9):946-952.

7. Kheirabadi GR, Maracy MR. Perinatal depression in a cohort study on Iranian women. J Res Med Sci. 2010;15(1):41-49.

8. Chandran M, Tharyan P, Muliyil J, Abraham S. Post-partum depression in a cohort of women from a rural area of Tamil Nadu, India. Incidence and risk factors. Br J Psychiatry. 2002;181:499-504.

9. Grantham-McGregor S, Cheung YB, Cueto S, Glewwe P, Richter L, Strupp B. Developmental potential in the first 5 years for children in developing countries. Lancet. 2007;369(9555):60-70.

10. Marcus S, Lopez JF, McDonough S, et al. Depressive symptoms during pregnancy: impact on neuroendocrine and neonatal outcomes. Infant Behav Dev. 2011;34(1):26-34.

11. Amorim P. Mini International Neuropsychiatric Interview (MINI): validation of brief interview to diagnose mental disorders. Rev Bras Psiquiatr. 2000;22(3):106-115. Portuguese.

12. Tenney NH, Schotte CK, Denys DA, van Megen HJ, Westenberg HG. Assessment of DSM-IV personality disorders in obsessive compulsive disorder: comparison of clinical diagnosis, self report questionnaire, and semi structured interview. J Pers Disord. 2003;17(6): 550-561.

13. Lima M. Avaliação dos níveis de ansiedade através da Escala de Hamilton em pacientes submetidos a psicoterapia breve grupal dinâmica associada a medicação e sem associação medicamentosa. [Evaluation of anxiety levels through the Hamilton Rating Scale in patients undergoing brief psychotherapy group dynamics associated with medication and without drug combination]. J Bras Psiquiatr. 1993;42:381-386. (Brazilian).

14. Fisher JR, Tran H, Tran T. Relative socioeconomic advantage and mood during advanced pregnancy in Vietnam. Int J Ment Health Syst. 2007; 1(1):3.

15. Pereira PK, Lovisi GM, Lima LA, Legay LF. Obstetric complications, stressful life events, violence and depression during pregnancy in adolescents at primary care setting. Rev Psiq Clín. 2010;37(5):216-222. Portuguese.

16. Engle PL, Black MM, Behrman JR, et al. Strategies to avoid the loss of developmental potential in more than 200 million children in the developing world. Lancet. 2007;369(9557):229-242.

17. Walker SP, Wachs TD, Gardner JM, et al. Child development: risk factors for adverse outcomes in developing countries. Lancet. 2007; 369(9556):145-157.

18. Forray A, Focseneanu M, Pittman B, McDougle CJ, Epperson CN. Onset and exacerbation of obsessive-compulsive disorder in pregnancy and the postpartum period. J Clin Psychiatry. 2010;71(8):1061-1068.

19. Rini CK, Dunkel-Schetter C, Wadhwa PD, Sandman CA. Psychological adaptation and birth outcomes: the role of personal resources, stress, and sociocultural context in pregnancy. Health Psychol. 1999;18(4): 333-345.

20. Glynn LM, Schetter CD, Hobel CJ, Sandman CA. Pattern of perceived stress and anxiety in pregnancy predicts preterm birth. Health Psychol. 2008;27(1):43-51.

21. Huizink AC, Robles de Medina PG, Mulder EJ, Visser GH, Buitelaar JK. Stress during pregnancy is associated with developmental outcome in infancy. J Child Psychol Psychiatry. 2003;44(6):810-818.

22. DiPietro JA, Novak MF, Costigan KA, Atella LD, Reusing SP. Maternal psychological distress during pregnancy in relation to child development at age two. Child Dev. 2006;77(3):573-587.

23. Kramer MS, Lydon J, Seguin L, et al. Stress pathways to spontaneous preterm birth: the role of stressors, psychological distress, and stress hormones. Am J Epidemiol. 2009;169(11):1319-1326.

24. Ji S, Long Q, Newport D, et al. Validity of depression rating scales during pregnancy and the postpartum period: impact of trimester and parity. J Psychiatr Res. 2011;45(2):213-219.

25. Murray D, Cox JL. Screening for depression during pregnancy with the Edinburgh Depression Scale (EDDS). J Reprod Infant Psychol. 1990;8(2): 99-107. 
26. Holcomb WL Jr, Stone LS, Lustman PJ, Gavard JA, Mostello DJ. Screening for depression in pregnancy: characteristics of the Beck Depression Inventory. Obstet Gynecol. 1996;88(6):1021-1025.

27. Marcus SM. Depression during pregnancy, rates, risks and consequences - Motherisk update 2008. Can J Clin Pharmacol. 2009;16(1): e15-e22.

28. Su KP, Chiu TH, Huang CL, et al. Different cutoff points for different trimesters? The use of Edinburgh Postnatal Depression Scale and Beck Depression Inventory to screen for depression in pregnant Taiwanese women. Gen Hosp Psychiatry. 2007;29(5):436-441.

29. Sahota PK, Jain SS, Dhand R. Sleep disorders in pregnancy. Curr Opin Pulm Med. 2003;9(6):477-483.

30. Parry BL, Martinez LF, Maurer EL, Lopez AM, Sorenson D, Meliska CJ. Sleep, rhythms and women's mood. Part 1 . Menstrual cycle, pregnancy and postpartum. Sleep Med Rev. 2006;10(2):129-144.

31. Altemus M, Fong J, Yang R, Damast S, Luine V, Ferguson D. Changes in cerebrospinal fluid neurochemistry during pregnancy. Biol Psychiatry. 2004;56(6):386-392.

32. McGuinness M, Blissett J, Jones C. OCD in the perinatal period: is postpartum OCD (ppOCD) a distinct subtype? A review of the literature. Behav Cogn Psychother. 2011;39(3):285-310.
33. Tandon SD, Cluxton-Keller F, Leis J, Le HN, Perry DF. A comparison of three screening tools to identify perinatal depression among lowincome African American women. J Affect Disord. August 22, 2011. [Epub ahead of print.]

34. Halbreich U, Karkun S. Cross-cultural and social diversity of prevalence of postpartum depression and depressive symptoms. J Affect Disord. 2006;91(2-3):97-111.

35. Gorenstein C, Andrade L. Beck Depression Inventory: psychometric properties of the Portuguese version. Rev Psiquiatr Clin. 1998;25: 245-250.

36. Da Costa D, Larouche J, Dritsa M, Brender W. Variations in stress levels over the course of pregnancy: factors associated with elevated hassles, state anxiety and pregnancy-specific stress. J Psychosom Res. 1999;47(6):609-621.

37. Gaynes BN, Gavin N, Meltzer-Brody S, et al. Perinatal depression: prevalence, screening accuracy, and screening outcomes. Evid Rep Technol Assess (Summ). 2005;(119):1-8.

\section{Publish your work in this journal}

Neuropsychiatric Disease and Treatment is an international, peerreviewed journal of clinical therapeutics and pharmacology focusing on concise rapid reporting of clinical or pre-clinical studies on a range of neuropsychiatric and neurological disorders. This journal is indexed on PubMed Central, the 'PsycINFO' database and CAS.
The manuscript management system is completely online and includes a very quick and fair peer-review system, which is all easy to use. Visit http://www.dovepress.com/testimonials.php to read real quotes from published authors.

Submit your manuscript here: http://www.dovepress.com/neuropsychiatric-disease-and-treatment-journal 